Tạp chí Cơ học Journal of Mechanics, NCNST of. Vietnam T. XVI, 1994, No $2(1-6)$

\title{
THE PROBLEM OF LONGITUDINAL SHOCK OF TWO SPHERICAL END ELASTIC BARS WITH VISCO-ELASTIC RESISTANCE FORCE
}

\author{
NGUYEN THUC AN, NGUYEN DANG TO, NGUYEN HUNG SON \\ Hanoi Water Resources University
}

Based on the theory of one-dimensional wave together with Dalembert solution and Hertz's law of deformation holds, in [1] and [2] we studied the problem of shock of two elastic bars with free spherical end. In this paper, we continue to study the above problem when the second end of the second bar meets visco-elastic resistance force.

\section{§1. FORMULATION OF THE PROBLEM}

The motion equation of the bars is:

$$
\frac{\partial^{2} U_{j}}{\partial t^{2}}=a_{j}^{2} \frac{\partial^{2} U_{j}}{\partial x_{j}^{2}}
$$

where $j=1,2 ; \quad a_{j}=\sqrt{\frac{E_{j}}{\rho_{j}}}$ - wave velocity.

Initial conditions: At $t=0$,

$$
\begin{aligned}
& \frac{\partial U_{1}^{\prime}}{\partial t}=V_{1} ; \quad U_{1}=0 ; \quad \frac{\partial U_{1}}{\partial x_{1}}=0 \\
& \frac{\partial U_{2}}{\partial t}=0 ; \quad U_{2}=0 ; \quad \frac{\partial U_{2}}{\partial x_{2}}=0
\end{aligned}
$$

Boundary conditions:

At the shock end $x_{1}=\ell_{1} ; x_{2}=\ell_{2}$,

$$
E_{1} F_{1} \frac{\partial U_{1}}{\partial x_{1}}=E_{2} F_{2} \frac{\partial U_{2}}{\partial x_{2}}=-K\left(U_{1}+U_{2}\right)^{3 / 2}
$$

At the free end, $x_{1}=0$,

$$
\frac{\partial U_{1}}{\partial x_{1}}=0
$$

When the end of the second bar bear on the visco-elastic sole, we obtain:

$$
x_{2}=0 ; \quad \frac{\partial U_{2}}{\partial x_{2}}=-K_{1} U_{2}-\lambda \frac{\partial U_{2}}{\partial t}
$$

In this equation $k_{1}, \lambda$ are elastic and viscid coefficients respectively. They are considered as constants. A general solution of eq. (1.1) is of the D'Alembert form:

$$
U_{j}=\varphi_{j}\left(a_{j} t-x_{j}\right)+\psi_{j}\left(a_{j} t+x_{j}\right)
$$




\section{§2. DETERMINATION OF WAVE FUNCTIONS OF BARS}

Assume that the second bar is in the rest, the first bar centro-longitudinally moves and impacts to the second one with velocity $V_{1}$, based on [1] we get:

$$
\begin{aligned}
& \varphi_{1}^{\prime}\left(z_{1}\right)=\frac{V_{1}}{2 a_{1}} \\
& \varphi_{2}^{\prime}\left(z_{2}\right)=0
\end{aligned}
$$

where $-\ell_{j}<z_{j}<\ell_{j}$, with $j=1,2$.

According to the boundary condition (1.3) we have:

$$
\begin{aligned}
\dot{A}\left(-\varphi_{2}^{\prime}+\psi_{2}^{\prime}\right)^{-1 / 3} \cdot\left(-\varphi_{2}^{\prime \prime}+\psi_{2}^{\prime \prime}\right) & =2 \varphi_{1}^{\prime}+B \varphi_{2}^{\prime}+C \psi_{2}^{\prime} \\
\psi_{1}^{\prime} & =\varphi_{1}^{\prime}+\frac{1}{\alpha}\left(-\varphi_{2}^{\prime}+\psi_{2}^{\prime}\right)
\end{aligned}
$$

where

$A=\frac{2}{3}\left(-\frac{1}{\beta}\right)^{2 / 3} \cdot\left(\frac{1}{\alpha}\right)^{2 / 3} \cdot \frac{a_{2}}{a_{1}} ; \quad B=\frac{\alpha a_{2}-a_{1}}{\alpha a_{1}} ; \quad C=\frac{\alpha a_{2}+a_{1}}{\alpha a_{1}} ; \quad \alpha=\frac{E_{1} \cdot F_{1}}{E_{2} \cdot F_{2}} ; \quad \beta=\frac{K}{E_{1} \cdot F_{1}}$, Consider that $\dot{T}_{2}=i T_{1}+q T_{1}$ with $i=1,2,3, \ldots ; 0 \leq q<1$.

The wave functions $\varphi_{1}^{\prime}\left(a_{1} t-x_{1}\right) ; \psi_{1}^{\prime}\left(a_{1} t+x_{1}\right) ; \varphi_{2}^{\prime}\left(a_{2} t-x_{2}\right)$ and $\psi_{2}^{\prime}\left(a_{2} t+x_{2}\right)$ with $0<t<T_{2}$ are determined as follows:

At first period $T_{1}\left(0<t<T_{1}\right)$, we have $\varphi_{2}^{\prime}=0$ and $\varphi_{2}^{\prime \prime}=0$, and from eq. (2.1) $\varphi_{1}^{\prime}=\frac{V_{1}}{2 a_{1}}$.

Notice that $\frac{d \psi_{2}}{d z}=\psi_{2}^{\prime}=y$ with $z=a_{2} t+\ell_{2}$ then eq. (2.2) can be written as follows:

$$
y^{\prime}=\frac{1}{A}\left(\frac{V_{1}}{a_{1}}+c y\right) \cdot y^{1 / 3}
$$

Integrated eq. (2.4) we have:

$$
z-z_{0}=\frac{3 A}{C_{1} \cdot C}\left[\frac{1}{2 \sqrt{3}} \ln \frac{y^{2 / 3}-C_{1} y^{1 / 3}+C_{1}^{2}}{\left(C_{1}+y^{1 / 3}\right)^{2}}+\operatorname{arctg} \frac{2 y^{1 / 3}-C_{1}}{\sqrt{3} C_{1}}-\operatorname{arctg}\left(-\frac{1}{\sqrt{3}}\right)\right]
$$

where $C_{1}=\frac{V_{1}}{C a_{1}}$. From eq. (2.3) we obtain:

$$
\psi_{1}^{\prime}=\frac{V_{1}}{2 a_{1}}+\frac{1}{\alpha} y
$$

Similarly [1] for the $i^{\text {th }}$ period of first bar we get:

$$
\begin{aligned}
\left(\varphi_{1}^{\prime}\right)_{1 i} & =\frac{Y_{1}}{2 a_{1}}+\frac{1}{\alpha} \sum_{n=1}^{i-1} y_{n} \\
y_{i}^{\prime} & =\frac{1}{A} y_{i}^{1 / 3} \cdot\left[\frac{V_{1}}{a_{1}}+\frac{2}{\alpha} \sum_{n=1}^{i-1} y_{n^{\prime}}+C y_{i}\right]
\end{aligned}
$$

Equation (2.7) can be solved by the finite difference method. Value $y_{i}$ of first bar at the start of period $i$ is equal to that one at the end of period $(i-1)$. From eq. (2.3) we have:

$$
\left(\psi_{1}^{\prime}\right)_{1 i}=\frac{V_{1}}{2 a_{1}}+\frac{1}{\alpha} \sum_{n=1}^{i} y_{n}
$$


Finally in the interval $i T_{1}^{*}<t<T_{2}=i T_{1}+q T_{1}$ we obtain:

$$
\varphi_{2}^{\prime}\left(a_{2} t-\ell_{2}\right)=0 \text { and } \varphi_{2}^{\prime \prime}\left(a_{2} t-\ell_{2}\right)=0 .
$$

From eq. (2.2) we have

$$
\left(\psi_{2}^{\prime \prime}\right)_{1}=\frac{1}{A}\left(\psi_{2}^{\prime}\right)_{1}^{1 / 3} \cdot\left[2\left(\varphi_{1}^{\prime}\right)_{1}+C\left(\psi_{2}^{\prime}\right)_{1}\right]
$$

where $\left(\varphi_{1}^{\prime}\right)_{1}=\left(\psi_{1}^{\prime}\right)_{1 i}$.

Solving eq. (2.9) the wave functions $\left(\psi_{2}^{\prime}\right)_{1}$ is determined. From eq. (2.3) we obtain:

$$
\left(\psi_{1}^{\prime}\right)_{1}=\left(\varphi_{1}^{\prime}\right)_{1}+\frac{1}{\alpha}\left(\psi_{2}^{\prime}\right)_{1}
$$

when $t<\frac{T_{2}}{2}$ reflected wave $\varphi_{2}^{\prime}\left(a_{2} t-x_{2}\right)$ does not appear in the second bar.

If $\frac{T_{2}}{2}<t<T_{2}$ then the wave function $\varphi_{2}^{\prime}\left(a_{2} t-\ell_{2}\right)=0$, but the wave function $\varphi_{2}^{\prime}\left(a_{2} t-x_{2}\right)$ appears in the second bar. Determination of the wave function $\varphi_{2}^{\prime}\left(a_{2} t-x_{2}\right)$ with $\frac{T_{2}}{2}<t<T_{2}$ is done in the same way described by [1]. According to boundary condition mentioned in eq. (1.5), the following cases are occured.

If $1-\lambda a_{2} \neq 0$ then:

$$
\varphi_{2}^{\prime}\left(a_{2} t\right)-\frac{K_{1}}{1-\lambda a_{2}} \varphi_{2}\left(a_{2} t\right)=\frac{1+\lambda a_{2}}{1-\lambda a_{2}} \psi_{2}^{\prime}\left(a_{2} t\right)+\frac{K_{1}}{1-\lambda a_{2}} \psi_{2}\left(a_{2} t\right)
$$

or

$$
\varphi_{2}^{\prime}\left(a_{2} t-x_{2}\right)-\frac{K_{1}}{1-\lambda a_{2}} \varphi_{2}\left(a_{2} t-x_{2}\right)=\frac{1+\lambda a_{2}}{1-\lambda a_{2}} \psi_{2}^{\prime}\left(a_{2} t-x_{2}\right)+\frac{K_{1}}{1-\lambda a_{2}} \psi_{2}\left(a_{2} t-x_{2}\right)
$$

with $0<a_{2} t-x_{2}<2 \ell_{2}$.

Based on eq. (2.1) when $0<a_{2} t-x_{2}<\ell_{2}$ then $\varphi_{2}^{\prime}\left(a_{2} t-x_{2}\right)=0$. When $\ell_{2}<a_{2} t-x_{2}<2 \ell_{2}$ then $\psi_{2}^{\prime}\left(a_{2} t-x_{2}\right)$ is known and $\psi_{2}\left(a_{2} t-x_{2}\right)$ is determined. Integrating eq. (2.11) with the condition of $\varphi_{2}\left(\ell_{2}-0\right)=0$ we obtain:

$$
\varphi_{2}\left(a_{2} t-x_{2}\right)=e^{-\frac{K_{1}}{1-\lambda a_{3}}\left(a_{2} t-x_{2}\right)} \cdot \int_{\ell_{2}}^{\left(a_{2} t-x_{2}\right)} e^{\frac{K_{1}}{1-\lambda a_{2}} \tau} \cdot\left[\frac{1+\lambda a_{2}}{1-\lambda a_{2}} \psi_{2}^{\prime}(\tau)+\frac{K_{1}}{1-\lambda a_{2}} \psi_{2}(\tau)\right] d \tau
$$

If $1-\lambda a_{2}=0$, we have:

$$
\varphi_{2}\left(a_{2} t-x_{2}\right)=-\frac{2}{K_{1}} \psi_{2}^{\prime}\left(a_{2} t-x_{2}\right)-\psi_{2}\left(a_{2} t-x_{2}\right)
$$

Based on those mentioned above, we can determine the wave function $\varphi_{2}^{\prime}\left(a_{2} t-x_{2}\right)$ in the second bar. So that we can determine wave function $\varphi_{1}^{\prime}\left(a_{1} t-x_{1}\right), \psi_{1}^{\prime}\left(a_{1} t+x_{1}\right), \varphi_{2}^{\prime}\left(a_{2} t-x_{2}\right)$ and $\psi_{2}^{\prime}\left(a_{2} t+x_{2}\right)$ at each of the sections of the bars in interval $0<t<\frac{2 \ell_{2}}{a_{2}}$.

In interval $T_{2}<t<2 T_{2}$ studying each of period $T_{1}$ with $T_{2}+(n-1) T_{1}<t<T_{2}+n T_{1}$. Let ()$_{2 n}$ be a wave function, that is determined in $n^{t h}$ period of first bar and the wave function itself is also determined in the second period of second bar, where $n=1,2, \ldots, i$. At the first period of first bar with $T_{2}<t<T_{2}+T_{1}$, according boundary condition (1.3) we have:

$$
\begin{aligned}
& \left(\psi_{2}^{\prime \prime}\right)_{21}=\left(\varphi_{2}^{\prime \prime}\right)_{21}+\frac{1}{A}\left[2\left(\varphi_{1}^{\prime}\right)_{21}+B\left(\varphi_{2}^{\prime}\right)_{21}+C\left(\psi_{2}^{\prime}\right)_{21}\right] \cdot\left[\left(-\varphi_{2}^{\prime}\right)_{21}+\left(\psi_{2}^{\prime}\right)_{21}\right]^{1 / 3} \\
& \left(\psi_{1}^{\prime}\right)_{21}=\left(\varphi_{1}^{\prime}\right)_{21}+\frac{1}{\alpha}\left[\left(-\varphi_{2}^{\prime}\right)_{21}+\left(\psi_{2}^{\prime}\right)_{21}\right]
\end{aligned}
$$


From condition (1.4)

$$
\varphi_{1}^{\prime}\left(a_{1} t-\ell_{1}\right)=\psi_{1}^{\prime}\left(a_{1} t-\ell_{1}\right)=\psi_{1}^{\prime}\left[a_{1}\left(t-T_{1}\right)+\ell_{1}\right]
$$

or

$$
\left(\varphi_{1}^{\prime}\right)_{21}=\left(\psi_{1}^{\prime}\right)_{20}
$$

where $\left(\psi_{1}^{\prime}\right)_{20}$ is the wave function $\psi_{1}^{\prime}\left(a_{1} t+\ell_{1}\right)$ with $\left(T_{2}-T_{1}\right)<t<T_{2}$, which was determined, so that the wave function $\left(\varphi_{1}^{\prime}\right)_{21}$ is also determined. According to (1.5) and (2.11) we have:

$$
\left(\varphi_{2}^{\prime}\right)_{21}-\frac{K_{1}}{1-\lambda a_{2}}\left(\varphi_{2}\right)_{21}=\frac{1+\lambda a_{2}}{1-\lambda a_{2}}\left(\psi_{2}^{\prime}\right)_{21}+\frac{K_{1}}{1-\lambda a_{2}}\left(\psi_{2}\right)_{11}
$$

If $1-\lambda a_{2} \neq 0$ then a solution of eq. (2.17) is:

$$
\left(\varphi_{2}\right)_{21}=e^{\frac{K_{1}}{1-\lambda a_{2}}\left(a_{2} t-\ell_{2}\right)} \cdot \int_{\ell_{2}}^{\left(a_{2} t-\ell_{2}\right)} e^{-\frac{K_{1}}{1-\lambda a_{2}} \tau} \cdot\left[\frac{1+\lambda a_{2}}{1-\lambda a_{2}} \psi_{2}^{\prime}(\tau)+\frac{K_{1}}{1-\lambda a_{2}} \psi_{2}(t)\right] d \tau
$$

If $1-\lambda a_{2}=0$ we have:

$$
\left(\varphi_{2}\right)_{21}=-\frac{2}{K_{1}}\left(\psi_{2}^{\prime}\right)_{11}-\left(\psi_{2}\right)_{11}
$$

So the wave function $\left(\varphi_{2}^{\prime}\right)_{21}=\left(\varphi_{2}^{\prime}\left(a_{2} t-\ell_{2}\right)\right)_{21}$ is known from eq. $(2.14)$ the function $\left(\psi_{2}^{\prime}\right)_{21}$ is determined. Replacing this result into eq. $(2.15)$ the wave function $\left(\psi_{1}^{\prime}\right)_{21}$ can be found. Doing similary we can determine the wave functions at the $i^{\text {th }}$ period of first bar. We have:

$$
\begin{aligned}
& \left(\psi_{2}^{\prime \prime}\right)_{2 i}=\left(\varphi_{2}^{\prime \prime}\right)_{2 i}+\frac{1}{A}\left[2\left(\varphi_{1}^{\prime}\right)_{2 i}+B\left(\varphi_{2}^{\prime}\right)_{2 i}+C\left(\psi_{2}^{\prime}\right)_{2 i}\right] \cdot\left[\left(-\varphi_{2}^{\prime}\right)_{2 i}+\left(\psi_{2}^{\prime}\right)_{2 i}\right]^{1 / 2} \\
& \left(\psi_{1}^{\prime}\right)_{2 i}=\left(\varphi_{1}^{\prime}\right)_{2 i}+\frac{1}{\alpha}\left[\left(-\varphi_{2}^{\prime}\right)_{2 i}+\left(\psi_{2}^{\prime}\right)_{2 i}\right] \\
& \left(\varphi_{1}^{\prime}\right)_{2 i}=\left(\psi_{1}^{\prime}\right)_{2(i-1)} \\
& \left(\varphi_{2}^{\prime}\right)_{2 i}-\frac{K_{1}}{1-\lambda a_{2}}\left(\varphi_{2}\right)_{2 i}=\frac{1+\lambda a_{2}}{1-\lambda a_{2}}\left(\psi_{2}^{\prime}\right)_{1 i}+\frac{K_{1}}{1-\lambda a_{2}}\left(\psi_{2}\right)_{1 i}
\end{aligned}
$$

If $1-\lambda a_{2} \neq 0$ then solution of eq. (2.23) is:

$$
\begin{aligned}
\left(\varphi_{2}\right)_{2 i} & =\left(\varphi_{2}\left(a_{2} t-\ell_{2}\right)\right)_{2 i}= \\
& =e^{\frac{K_{1}}{1-\lambda a_{3}}\left(a_{2} t-\ell_{2}\right)} \cdot\left\{\int_{\left[\ell_{2}+a_{2}(i-1) T_{1}\right]}^{\left(a_{2} t-\ell_{2}\right)} e^{-\frac{K_{1}}{1-\lambda a_{2}} \tau} \cdot\left[\frac{1+\lambda a_{2}}{1-\lambda a_{2}} \psi_{2}^{\prime}(\tau)+\frac{K_{1}}{1-\lambda a_{2}} \psi_{2}(\tau)\right] d \tau+C_{2 i}\right\}
\end{aligned}
$$

where

$$
C_{2 i}=\varphi_{2}\left[\ell_{2}+a_{2}(i-1) T_{1}-0\right] \cdot e^{-\frac{K_{1}}{i-\lambda a_{2}}\left[\ell_{2}+a_{2}(i-1) T_{1}\right]}
$$

If $1-\lambda a_{2}=0$ then

$$
\left(\varphi_{2}\right)_{2 i}=-\frac{2}{K_{1}}\left(\psi_{2}^{\prime}\right)_{1 i}-\left(\psi_{2}\right)_{1 i}
$$

So that the wave functions $\left(\psi_{2}^{\prime}\right)_{2 i}$ and $\left(\psi_{1}^{\prime}\right)_{2 i}$ are determined. Now we determine the wave functions is odd part of the second period of the second bar. From conditions (1.3), (1.4) and (1.5) we have:

$$
\begin{aligned}
& \left(\psi_{2}^{\prime \prime}\right)_{2}=\left(\varphi_{2}^{\prime \prime}\right)_{2}+\frac{1}{A}\left[2\left(\varphi_{1}^{\prime}\right)_{2}+B\left(\varphi_{2}^{\prime}\right)_{2}+C\left(\psi_{2}^{\prime}\right)_{2}\right] \cdot\left[\left(-\varphi_{2}^{\prime}\right)_{2}+\left(\psi_{2}^{\prime}\right)_{2}\right]^{1 / 3} \\
& \left(\psi_{1}^{\prime}\right)_{2}=\left(\varphi_{1}^{\prime}\right)_{2}+\frac{1}{\alpha}\left[\left(-\varphi_{2}^{\prime}\right)_{2}+\left(\psi_{2}^{\prime}\right)_{2}\right] \\
& \left(\varphi_{1}^{\prime}\right)_{2}=\left(\psi_{1}^{\prime}\right)_{2 i} \\
& \left(\varphi_{2}^{\prime}\right)_{2}-\frac{K_{1}}{1-\lambda a_{2}}\left(\varphi_{2}\right)_{2}=\frac{1+\lambda a_{2}}{1-\lambda a_{2}}\left(\psi_{2}^{\prime}\right)_{1}+\frac{K_{1}}{1-\lambda a_{2}}\left(\psi_{2}\right)_{1}
\end{aligned}
$$


If $1-\lambda a_{2} \neq 0$ then

$$
\left(\varphi_{2}\right)_{2}=e^{\frac{K_{1}}{1-\lambda a_{2}}\left(a_{2} t-\ell_{2}\right)} \cdot\left\{\int_{\ell_{3}+a_{2} i T_{1}}^{a_{2} t-\ell_{2}} e^{-\frac{\kappa_{1}}{1-\lambda a_{2}} \tau} \cdot\left[\frac{1+\lambda a_{2}}{1-\lambda a_{2}} \psi_{2}^{\prime}(\tau)+\frac{K_{1}}{1-\lambda a_{2}} \psi_{2}(\tau)\right] d \tau+C_{2}\right\}
$$

where

$$
C_{2}=\varphi_{2}\left(\ell_{2}+a_{2} i T_{1}-0\right) e^{-\frac{K_{1}}{1-\lambda a_{2}}\left(\ell_{3}+a_{2} i T_{1}\right)}
$$

If $1-\lambda a_{2}=0$ then

$$
\left(\varphi_{2}\right)_{2}=-\frac{2}{K_{1}}\left(\psi_{2}^{\prime}\right)_{1}-\left(\psi_{2}\right)_{1}
$$

So that the wave functions $\left(\psi_{2}^{\prime}\right)_{2}$ and $\left(\psi_{1}^{\prime}\right)_{2}$ are determined. If the shocks of two bare are still not finished yet in second period of second bar, the next periods are studied is the same method as above mentioned. Let ()$_{p n}$ be the wave function in $n^{\text {th }}$ period of first and in $p^{\text {th }}$ period of second bar. In interval $(p-1) T_{2}+(n-1) T_{1}<t<(p-1) T_{2}+n T_{1}$ with $n=1,2, \ldots$, the problem is studied as following:

From conditions (1.3) and (1.4) we have:

$$
\begin{aligned}
\left(\psi_{2}^{\prime \prime}\right)_{p n} & =\left(\varphi_{2}^{\prime \prime}\right)_{p n}+\frac{1}{A}\left[2\left(\varphi_{1}^{\prime}\right)_{p n}+B\left(\varphi_{2}^{\prime}\right)_{p n}+C\left(\psi_{2}^{\prime}\right)_{p n}\right] \cdot\left[\left(-\varphi_{2}^{\prime}\right)_{p n}+\left(\psi_{2}^{\prime}\right)_{p n}\right]^{1 / 3} \\
\left(\psi_{1}^{\prime}\right)_{p n} & =\left(\varphi_{1}^{\prime}\right)_{p n}+\frac{1}{\alpha}\left[\left(-\varphi_{2}^{\prime}\right)_{p n}+\left(\psi_{2}^{\prime}\right)_{p n}\right] \\
\left(\varphi_{1}^{\prime}\right)_{p n} & =\left(\psi_{1}^{\prime}\right)_{p(n-1)}
\end{aligned}
$$

By simillar way mentioned above, and from condition (1.5) we obtain:

If $1-\lambda a_{2} \neq 0$ then

$$
\left(\varphi_{2}\right)_{p n}=e^{\frac{K_{1}}{1-\lambda a_{2}}\left(a_{2} t-\ell_{2}\right)} \cdot\left\{\int_{a_{2}\left[(2 p-3) \frac{T_{2}}{2}+(n-1) T_{1}\right]}^{a_{3} t-\ell_{2}} e^{-\frac{K_{1}}{1-\lambda a_{2}} \tau} \cdot\left[\frac{1+\lambda a_{2}}{1-\lambda a_{2}} \psi_{2}^{\prime}(\tau)+\frac{K_{1}}{1-\lambda a_{2}} \psi_{2}(\tau)\right] d \tau+\dot{C}_{p n}\right\}
$$

where $p \geq 2$, and

$$
C_{p n}=\varphi_{2}\left[a_{2}(2 p-3) \frac{T_{2}}{2}+a_{2}(n-1) T_{1}-0\right] \cdot e^{-\frac{K_{1}}{1-\lambda a_{2}}\left[a_{2}(2 p-3) \frac{T_{2}}{2}+a_{3}(n-1) T_{1}\right]} .
$$

If $1-\lambda a_{2}=0$ then

$$
\left(\varphi_{2}\right)_{p n}=-\frac{2}{K_{1}}\left(\psi_{2}^{\prime}\right)_{(p-1) n}-\left(\psi_{2}\right)_{(p-1) n}
$$

So that the wave functions $\left(\psi_{2}^{\prime}\right)_{p n}$ and $\left(\psi_{1}\right)_{p n}$ are determined. Now we are studying this problem in final odd part of the $p^{t h}$ period of the second bar, or $(p-1) T_{2}+i T_{1}<t<(p-1) T_{2}+i T_{1}+q T_{1}=p T_{2}$. Let ()$_{p}$ be wave function determined in odd part of $p^{\text {th }}$ period of the second bar. From condition (1.3) and (1.4) we have

$$
\begin{aligned}
& \left(\psi_{2}^{\prime \prime}\right)_{p}=\left(\varphi_{2}^{\prime \prime}\right)_{p}+\frac{1}{A}\left[2\left(\varphi_{1}^{\prime}\right)_{p}+B\left(\varphi_{2}^{\prime}\right)_{p}+C\left(\psi_{2}^{\prime}\right)_{2}\right] \cdot\left[\left(-\varphi_{2}^{\prime}\right)_{p}+\left(\psi_{2}^{\prime}\right)_{p}\right]^{1 / 3} \\
& \left(\psi_{1}^{\prime}\right)_{p}=\left(\varphi_{1}^{\prime}\right)_{p}+\frac{1}{\alpha}\left[\left(-\varphi_{2}^{\prime}\right)_{p}+\left(\psi_{2}^{\prime}\right)_{p}\right] \\
& \left(\varphi_{1}^{\prime}\right)_{p}=\left(\psi_{1}^{\prime}\right)_{p i}
\end{aligned}
$$


Doing similarly, from eq. (1.5) we get:

If $1-\lambda a_{2} \neq 0$ then

$$
\left(\varphi_{2}\right)_{p}=e^{\frac{K_{2}}{1-\lambda a_{2}}\left(a_{2} t-\ell_{2}\right)} \cdot\left\{\int_{a_{2}\left\lfloor(2 p-3) \frac{T_{2}}{3}+i T_{1}\right]}^{a_{2} t-\ell_{2}} e^{-\frac{K_{1}}{1-\lambda a_{2}} \tau} \cdot\left[\frac{1+\lambda a_{2}}{1-\lambda a_{2}} \psi_{2}^{\prime}(\tau)+\frac{K_{1}}{1-\lambda a_{2}} \psi_{2}(\tau)\right] d \tau+C_{p}\right\}
$$

where

$$
C_{p}=\varphi_{2}\left[a_{2}(2 p-3) \frac{T_{2}}{2}+i a_{2} T_{1}-0\right] \cdot e^{-\frac{K_{1}}{1-\lambda a_{3}}\left(a_{2}(2 p-3) \frac{T_{2}}{2}+a_{2} i T_{1}\right.} .
$$

If $1-\lambda a_{2}=0$ then

$$
\left(\varphi_{2}\right)_{p}=-\frac{2}{K_{1}}\left(\psi_{2}^{\prime}\right)_{(p-1)}-\left(\psi_{2}\right)_{(p-1)}
$$

So that the wave functions $\left(\psi_{2}^{\prime}\right)_{p}$ and $\left(\psi_{1}^{\prime}\right)_{p}$ are determined. Impact-pressing force $F$ between two bars is determined by the following expression: $(F)_{p n}=E_{2} F_{2}\left[\left(-\varphi_{2}^{\prime}\right)_{p n}+\left(\psi_{2}^{\prime}\right)_{p n}\right]$ and $(F)_{p}=E_{2} F_{2}\left[\left(-\varphi_{2}^{\prime}\right)_{P}+\left(\psi_{2}^{\prime}\right)_{p}\right]$. Impact time determined by the following expression $(F)_{p n}=0$, or $(F)_{p}=0$. So we can determine the wave functions $\varphi_{1}^{\prime}\left(a_{1} t-x_{1}\right), \psi_{1}^{\prime}\left(a_{1} t+x_{1}\right), \varphi_{2}^{\prime}\left(a_{2} t-x_{2}\right)$ and $\psi_{2}^{\prime}\left(a_{2} t+x_{2}\right)$ at each section of two bars in impact time, and whence stress, velocity in each section of bars can be found.

\section{§3. CONCLUSION}

In this paper the authors have studied the problem of longitudinal shock of two spherical end elastic bars with visco-elastic resistance force. The wave function, stress, velocity in each section of bars, impact-pressing force between two bàrs and impact time are given. The considered model can be applied for pile driving on visco-elastic soil.

This publication is completed with financial support from the National Basic Research Program in Natural Sciences.

\section{REFERENCES}

1. Nguyen Thuc An, Nguyen Dang To and Nguyen Hung Son. Shock of two elastic bars with free spherical end. Journal of Mechanics, No 1, 1993.

2. Nguyen Thuc An, Nguyen Dang To and Nguyen Hung Son. Shock of two elastic bars with free spherical end. Journal of Mechanics, No 3, 1993.

3. Kiltevxki N. A. The theory of shock of solid bodies. Kiev 1969.

Received November 16, 1999

\section{VA CHẠM DỌC CỦA HAI THANH ĐẦU HÌNH CẪU VớI LỰC CẢN ĐAN NHỚT}

Trong bài báo này các tác giả xét bài toán va chạm dọc của hai thanh đàn hồi đầu hình cầu với đầu kia của thanh thứ hai gặp lực cản đàn nhớt. Đã xác định được hàm sóng, tì̀ đó xác định được ứng suất, vận tốc tại mồi thiết diện của thanh, lực nén va chạm giữa hai thanh và thời gian va chạm. 